\title{
Exact Solutions for Nonlinear Wave Equations by the Exp-Function Method
}

\author{
Mingsheng Hu, ${ }^{1,2}$ Zhijuan Jia, ${ }^{3}$ Qiaoling Chen, ${ }^{1}$ and Suiming Jia ${ }^{1}$ \\ ${ }^{1}$ College of Information Science \& Technology, Zhengzhou Normal University, Zhengzhou 450044, China \\ ${ }^{2}$ Institute of Systems Engineering, Huazhong University of Science \& Technology, Wuhan 430074, China \\ ${ }^{3}$ College of Computer, Wuhan University of Technology, Wuhan 430070, China
}

Correspondence should be addressed to Zhijuan Jia; jzj523@163.com and Qiaoling Chen; chenqiaoling007@163.com

Received 14 March 2014; Revised 20 April 2014; Accepted 21 April 2014; Published 6 May 2014

Academic Editor: Xiao-Jun Yang

Copyright (C) 2014 Mingsheng Hu et al. This is an open access article distributed under the Creative Commons Attribution License, which permits unrestricted use, distribution, and reproduction in any medium, provided the original work is properly cited.

This paper elucidates the main advantages of the exp-function method in finding exact solutions of nonlinear wave equations. By the aid of some mathematical software, the solution process becomes extremely simple and accessible.

\section{Introduction}

One of the most important aspects in nonlinear science is how to solve an exact solution of a nonlinear equation. Recently many different methods have appeared, among which the homotopy perturbation method [1-4], the tanhmethod [5], the sinh-method [6,7], and the F-expansion method [8-11] have caught much attention; however, all these methods are valid for some special kinds of nonlinear equations. It is therefore very much needed to find a universal approach to nonlinear equations; this is very challenging indeed, and the exp-function method [12-15] meets this requirement. The exp-function method itself is mathematically beautiful and extremely accessible to nonmathematicians. The use of the method requires no special knowledge of advanced calculus, and it is especially effective for solitary solutions.

\section{Exp-Function Method}

The exp-function method was first proposed by $\mathrm{He}$ and $\mathrm{Wu}$ [16], and we consider a general partial differential equation (PED) in the form

$$
P\left(u, u_{t}, u_{x}, u_{y}, u_{x x}, u_{t t}, u_{y y}\right)=0
$$

to pick out the main solution process and its advantages.
Use a transformation [16]

$$
\xi=k x+\omega t+l y,
$$

where $k, \omega$, and $l$ are unknown constants and should be determined later. By (2), we can convert (1) to the following nonlinear ordinary differential equation:

$$
G\left(u, u^{\prime}, u^{\prime \prime}, u^{\prime \prime \prime}, \ldots\right)=0
$$

According to the exp-function method, we assume that its solution can be expressed in the following form $[16,17]$ :

$$
u(\xi)=\frac{\sum_{n=-c}^{d} a_{n} \exp (n \xi)}{\sum_{m=-p}^{q} b_{n} \exp (m \xi)},
$$

where $c, d, p, q$ are positive integers that could be freely chosen. To determine the value of $c$ and $p$, we balance the linear term of highest order of (3) with the highest order of the nonlinear term. Similarly for determining the value of $d$ and $q$, we balance the lowest orders of linear and nonlinear terms in (3). By substituting (4) into (3), collecting terms of the same term of $\exp (i \xi)$, and equating the coefficient of each power of exp to zero, we can get a set of algebraic equations for determining unknown constants. 


\section{Exact Solution for Nonlinear Wave Equation}

In order to illustrate the basic solution process of the expfunction method, we use the Burgers-Huxley equation as an example, which can be expressed as [18]

$$
u_{t}+u_{x x}+\frac{3}{k} u u_{x}+c u+u^{2}+u^{3}=0
$$

where $u=u(x, t)$ is an unknown function, $u_{t}, u_{x}$ are the partial derivatives of $u(x, t)$ with respect to $t$ and $x$, respectively, and $k$ and $c$ are arbitrary constants.

According to the exp-function method [15-17], we introduce a complex variation $\xi$ defined as

$$
\xi=k x+\omega t
$$

Equation (5) thus becomes an ordinary differential equation as

$$
k^{2} u^{\prime \prime}+(3 u+\omega) u^{\prime}+c u+u^{2}+u^{3}=0
$$

We suppose that the solution of (7) can be expressed as

$$
u(\xi)=\frac{a_{c} \exp (c \xi)+\cdots+a_{-d} \exp (-d \xi)}{b_{p} \exp (p \xi)+\cdots+b_{-q} \exp (-q \xi)}
$$

Thus we have

$$
\begin{gathered}
u^{\prime \prime}=\frac{\gamma_{1} \exp ((c+3 p) \xi)}{\gamma_{2} \exp (4 \xi)} \\
u^{3}=\frac{c_{3} \exp (3 c \xi)+\cdots}{c_{4} \exp (3 p \xi)+\cdots}=\frac{c_{3} \exp ((3 c+p) \xi)}{c_{4} \exp (4 p \xi)} .
\end{gathered}
$$

Balancing highest order of exp-function in (9), we have $3 c+$ $p=c+3 p$, which leads to the result $p=c$. Similarly we balance the lowest orders of linear and nonlinear terms in (5) to determine values of $d$ and $q$, and we can get $d=q$. For simplicity, we set $p=c=1$ and $q=d=1$; then (8) reduces to

$$
u(\xi)=\frac{a_{1} \exp (\xi)+a_{0}+a_{-1} \exp (-\xi)}{\exp (\xi)+b_{0}+b_{-1} \exp (-\xi)}
$$

Substituting (10) in to (5), we have

$$
\begin{aligned}
& \frac{1}{A}\left[E_{3} \exp (3 \xi)+E_{2} \exp (2 \xi)+E_{1} \exp (\xi)+E_{0}\right. \\
& \left.\quad+E_{-1} \exp (-\xi)+E_{-2} \exp (-2 \xi)+E_{-3} \exp (-3 \xi)\right]=0
\end{aligned}
$$

where $=\left[b_{0}+\exp (\xi)+b_{-1} \exp (-\xi)\right]^{3}$,

$$
\begin{aligned}
& E_{3}= a_{1}^{3}+a_{1}^{2}+c a_{1}, \\
& E_{2}= c a_{0}-a_{0} a_{1}-\omega a_{0}+3 a_{0} a_{1}^{2}+4 a_{1}^{2} b_{0} \\
&+k^{2} a_{0}+2 a_{1} b_{0} c+\omega a_{1} b_{0}-k^{2} a_{1} b_{0} \\
& E_{0}= a_{0}^{3}+a_{0}^{2} b_{0}-7 a_{0} a_{-1}+6 a_{0} a_{1} a_{-1} \\
&+11 a_{0} a_{1} b_{-1}+2 b_{0} a_{1} a_{-1}+2 c a_{0} b_{-1}+2 c b_{0} a_{-1} \\
&-3 \omega b_{0} a_{-1}+c a_{0} b_{0}^{2}-6 k^{2} a_{0} b_{-1}+3 k^{2} a_{-1} b_{0} \\
&+3 k^{2} a_{-1} b_{-1} b_{0}+2 c a_{1} b_{-1} b_{0}+3 \omega a_{1} b_{-1} b_{0}, \\
& E_{1}= c a_{-1}-4 a_{1} a_{-1}-2 \omega a_{-1}+3 a_{1} a_{0}^{2}+3 a_{1}^{2} a_{-1} \\
&+7 a_{1}^{2} b_{-1}+4 k^{2} a_{-1}-2 a_{0}^{2}+\left(k^{2}+c\right) a_{1} b_{0}^{2}+5 a_{0} a_{1} b_{0} \\
&+2 c a_{1} b_{-1}-\omega a_{0} b_{0}+2 \omega a_{1} b_{-1}+2 c a_{0} b_{0}, \\
& E_{-2}=3 a_{0} a_{-1}^{2}-2 a_{-1}^{2} b_{0}+k^{2} a_{0} b_{-1}^{2}+5 a_{0} a_{-1} b_{-1} \\
&+(c+\omega) a_{0} b_{-1}^{2}+\left(2 c-k^{2}-\omega\right) a_{-1} b_{-1} b_{0}, \\
& E_{-1}=3 a_{0}^{2} a_{-1}+3 a_{1} a_{-1}^{2}+4 a_{0}^{2} b_{-1}-5 a_{-1}^{2}+a_{-1} b_{0}^{2} k^{2} \\
&+ 4 a_{1} b_{-1}^{2} k^{2}-a_{0} a_{-1} b_{0}+8 a_{1} a_{-1} b_{-1}+(2 c-2 \omega) a_{-1} b_{-1} \\
&+c a_{-1} b_{0}^{2}+c a_{1} b_{-1}^{2}-4 a_{-1} b_{-1}^{2} k^{2}-\omega a_{-1} b_{0}^{2}+2 \omega a_{1} b_{-1}^{2} \\
&\left(2 c+\omega-k^{2}\right) a_{0} b_{-1} b_{0}, \\
&(c)
\end{aligned}
$$$$
E_{-3}=a_{-1}^{3}+a_{-1}^{2} b_{-1}+c a_{-1} b_{-1}^{2} \text {. }
$$

Setting the coefficients of $\exp (i \xi),(i=0, \pm 1, \pm 2, \pm 3)$ to zero, we have

$$
\begin{array}{rlrl}
E_{3}=0, & E_{2}=0, & E_{1}=0 \\
E_{0}=0, & \\
E_{-3}=0, & E_{-2}=0, & E_{-1}=0 .
\end{array}
$$

With the help of some mathematical software, we can solve the solutions of the algebraic equations.

Case 1. Consider

$$
\begin{gathered}
a_{1}=\frac{\sqrt{1-4 c}-1}{2}, \\
b_{0}=\frac{-a_{0}\left(3 a_{1}^{2}-a_{1}+k^{2}+c-\omega\right)}{a_{1}\left(-k^{2}+4 a_{1}+2 c+\omega\right)},
\end{gathered}
$$

$$
\begin{gathered}
\omega=\frac{c a_{0}-a_{0} a_{1}+3 a_{0} a_{1}^{2}+4 a_{1}^{2} b_{0}+a_{0} k^{2}+2 a_{1} b_{0} c-a_{1} b_{0} k^{2}}{a_{0}-a_{1} b_{0}}, \\
a_{-1}=\frac{b_{-1}(\sqrt{1-4 c}-1)}{2} .
\end{gathered}
$$


This implies the following exact solution:

$$
\begin{aligned}
u(\xi)= & \left(\frac{\sqrt{1-4 c}-1}{2} \exp (\xi)+a_{0}\right. \\
& \left.+\frac{b_{2}(\sqrt{1-4 c}-1)}{2} \exp (-\xi)\right) \\
& \times\left(\exp (\xi)-\frac{\left(3 a_{0} a_{1}^{2}-a_{0} a_{1}+a_{0} k^{2}+c a_{0}-\omega a_{0}\right)}{2 c a_{1}+\omega c-a_{1} k^{2}+4 a_{1}^{2}}\right. \\
& \left.\quad+b_{2} \exp (-\xi)\right)^{-1},
\end{aligned}
$$

where

$$
\begin{aligned}
\xi= & k x \\
& +\frac{c a_{0}-a_{0} a_{1}+3 a_{0} a_{1}^{2}+4 a_{1}^{2} b_{0}+a_{0} k^{2}+2 a_{1} b_{0} c-a_{1} b_{0} k^{2}}{a_{0}-a_{1} b_{0}} t,
\end{aligned}
$$

$a_{0}, b_{2}$ are parameters, $b_{2} \neq 0$, and $k$ is a free real number.

Case 2. Consider

$$
\begin{gathered}
a_{1}=\frac{1}{2}, \quad b_{0}=0, \\
a_{2}=-\frac{b_{2}}{2}, \quad b_{2}=b_{2}, \\
a_{0}=\frac{\sqrt{2}}{2}+\sqrt{-16 b_{2} k^{2}+\frac{19}{2} b_{2}+2 c b_{2}+8 b_{2} \omega}, \\
\omega=k^{2}+c+\frac{1}{4} .
\end{gathered}
$$

This case gives another exact solution as follows:

$$
\begin{aligned}
u(x, t)=( & \frac{1}{2} \exp \left(k x+\left(k^{2}+c+\frac{1}{4}\right) t\right) \\
& +\frac{\sqrt{2}}{2} \sqrt{10 b_{2} c-8 b_{2} k^{2}+\frac{19}{2} b_{2}+2 b_{2}} \\
& \left.-b_{2} \exp \left(-k x-\left(k^{2}+c+\frac{1}{4}\right) t\right)\right) \\
& \times\left(\exp \left(k x+\left(k^{2}+c+\frac{1}{4}\right) t\right)\right. \\
& \left.+b_{2} \exp \left(-k x-\left(k^{2}+c+\frac{1}{4}\right) t\right)\right)^{-1},
\end{aligned}
$$

where $b_{2}, k$, are nonzero free parameters.
Case 3. Consider

$$
\begin{gathered}
a_{1}=-\frac{\sqrt{1-4 c}+1}{2}, \quad a_{0}=0, \\
b_{0}=0, \quad b_{2}=b_{2}, \quad a_{2}=-\frac{(1+\sqrt{1-4 c})}{2} b_{2} .
\end{gathered}
$$

This results in the following exact solution:

$$
\begin{aligned}
u(\xi)= & \left(-\frac{\sqrt{1-4 c}+1}{2} \exp (\xi)\right. \\
& \left.-\frac{(1+\sqrt{1-4 c})}{2} b_{2} \exp (-\xi)\right) \\
& \times\left(\exp (\xi)+b_{2} \exp (-\xi)\right)^{-1}
\end{aligned}
$$

where $b_{2}$ is nonzero free parameter.

\section{Conclusion}

By some mathematical software, the solution process is extremely simple and abundant solutions are predicted [1921]. The exp-function method is a universal tool for nonlinear equations and can be easily extended to fractional calculus [22-27].

\section{Conflict of Interests}

The authors declare that there is no conflict of interests regarding the publication of this paper.

\section{Acknowledgments}

This project is sponsored by the National Natural Science Foundation of China (NSFC, Grants U1204703 and U1304614), the Key Scientific and Technological Project of Henan Province (122102310004), the Fundamental Research Funds for the Central Universities (HUST: 2012QN087 and 2012QN088), and the Innovation Scientists and Technicians Troop Construction Projects of Zhengzhou City (10LJRC190).

\section{References}

[1] J.-H. He, "Application of homotopy perturbation method to nonlinear wave equations," Chaos, Solitons and Fractals, vol. 26, no. 3, pp. 695-700, 2005.

[2] Y. S. Yun and C. Temuer, "Homotopy perturbation method for viscous heating in plane couette flow," Thermal Science, vol. 17, no. 5, pp. 1355-1360, 2013.

[3] L. M. Yan, "Modified homotopy perturbation method coupled with laplace transform for fractional heat transfer and porous media equations," Thermal Science, vol. 17, no. 5, pp. 1409-1414, 2013.

[4] J. H. He, "Homotopy perturbation method with two expanding parameters," Indian Journal of Physics, vol. 88, no. 2, pp. 193-196, 2014. 
[5] A.-M. Wazwaz, "The tanh method: exact solutions of the sineGordon and the sinh-Gordon equations," Applied Mathematics and Computation, vol. 167, no. 2, pp. 1196-1210, 2005.

[6] A. M. Wazwaz, "Exact solutions to the double sinh-Gordon equation by the tanh method and a variable separated ODE method," Computers \& Mathematics with Applications, vol. 50, no. 10-12, pp. 1685-1696, 2005.

[7] F. Pedit and H. Wu, "Discretizing constant curvature surfaces via loop group factorizations: the discrete sine- and sinhGordon equations," Journal of Geometry and Physics, vol. 17, no. 3, pp. 245-260, 1995.

[8] A. Ebaid and E. H. Aly, "Exact solutions for the transformed reduced Ostrovsky equation via the F-expansion method in terms of Weierstrass-elliptic and Jacobian-elliptic functions," Wave Motion, vol. 49, no. 2, pp. 296-308, 2012.

[9] M. T. Darvishi, M. Najafi, and M. Najafi, "Traveling wave solutions for the $(3+1)$-dimensional breaking soliton equation by $\left(G^{\prime} / G\right)$-expansion method and modified F-expansion method," International Journal of Computational and Mathematical Sciences, vol. 6, no. 2, pp. 64-69, 2012.

[10] Y. He, "New Jacobi elliptic function solutions for the Kudryashov-Sinelshchikov equation using improved F-expansion method," Mathematical Problems in Engineering, vol. 2013, Article ID 104894, 6 pages, 2013.

[11] A. Bhrawy and M. Obaid, "New exact solutions for the ZhiberShabat equation using the extended F-expansion method," Life Science Journal, vol. 9, no. 4, pp. 1154-1162, 2012.

[12] J.-H. He and M. A. Abdou, "New periodic solutions for nonlinear evolution equations using Exp-function method," Chaos, Solitons \& Fractals, vol. 34, no. 5, pp. 1421-1429, 2007.

[13] J.-H. He, "New interpretation of homotopy perturbation method," International Journal of Modern Physics B: Condensed Matter Physics, Statistical Physics, Applied Physics, vol. 20, no. 18, pp. 2561-2568, 2006.

[14] J. Liu, H.-Y. Luo, G. Mu et al., "New multi-soliton solutions for generalized Burgers-Huxley equation," Thermal Science, vol. 17, no. 5, pp. 1486-1489, 2013.

[15] J.-H. He, "Asymptotic methods for solitary solutions and compactons," Abstract and Applied Analysis, vol. 2012, Article ID 916793, 130 pages, 2012.

[16] J.-H. He and X.-H. Wu, "Exp-function method for nonlinear wave equations," Chaos, Solitons \& Fractals, vol. 30, no. 3, pp. 700-708, 2006.

[17] J.-H. He, "Exp-function method for fractional differential equations," International Journal of Nonlinear Sciences and Numerical Simulation, vol. 14, no. 6, pp. 363-366, 2013.

[18] N. A. Kudryashov and N. B. Loguinova, "Extended simplest equation method for nonlinear differential equations," Applied Mathematics and Computation, vol. 205, no. 1, pp. 396-402, 2008.

[19] H. Naher, F. A. Abdullah, and M. Ali Akbar, "New traveling wave solutions of the higher dimensional nonlinear partial differential equation by the exp-function method," Journal of Applied Mathematics, vol. 2012, Article ID 575387, 14 pages, 2012.

[20] X.-W. Zhou, "Exp-function method for solving Huxley equation," Mathematical Problems in Engineering, vol. 2008, Article ID 538489, 7 pages, 2008.

[21] H.-Z. Liu, "A simplification for Exp-function method when the balanced nonlinear term is a certain product," Abstract and Applied Analysis, vol. 2013, Article ID 264549, 5 pages, 2013.
[22] X. J. Yang and D. Baleanu, "Fractal heat conduction problem solved by local fractional variation iteration method," Thermal Science, vol. 17, no. 2, pp. 625-628, 2013.

[23] X.-J. Yang, H. M. Srivastava, J.-H. He, and D. Baleanu, "Cantortype cylindrical-coordinate method for differential equations with local fractional derivatives," Physics Letters: A, vol. 377, no. 28-30, pp. 1696-1700, 2013.

[24] W. H. Su, D. Baleanu, X. J. Yang et al., "Damped wave equation and dissipative wave equation in fractal strings within the local fractional variational iteration method," Fixed Point Theory and Applications, vol. 2013, article 89, 2013.

[25] X. J. Yang, D. Baleanu, M. P. Lazarevi et al., "Fractal boundary value problems for integral and differential equations with local fractional operators," Thermal Science, p. 103, 2013.

[26] M. Li, S. C. Lim, C. Cattani, and M. Scalia, "Characteristic roots of a class of fractional oscillators," Advances in High Energy Physics, vol. 2013, Article ID 853925, 7 pages, 2013.

[27] M. Li, "A class of negatively fractal dimensional Gaussian random functions," Mathematical Problems in Engineering, vol. 2011, Article ID 291028, 18 pages, 2011. 


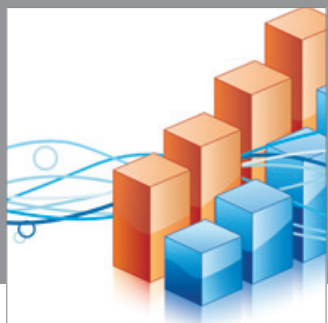

Advances in

Operations Research

mansans

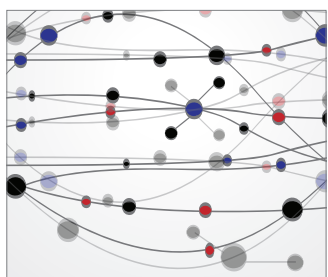

The Scientific World Journal
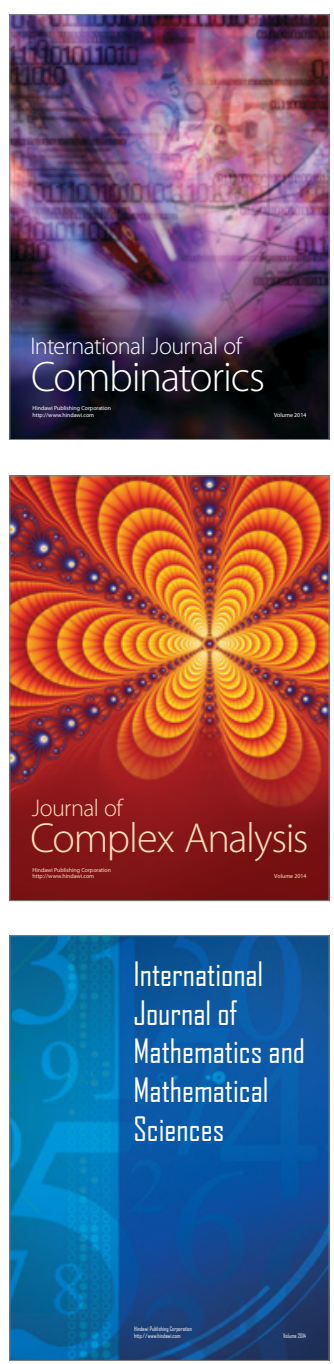
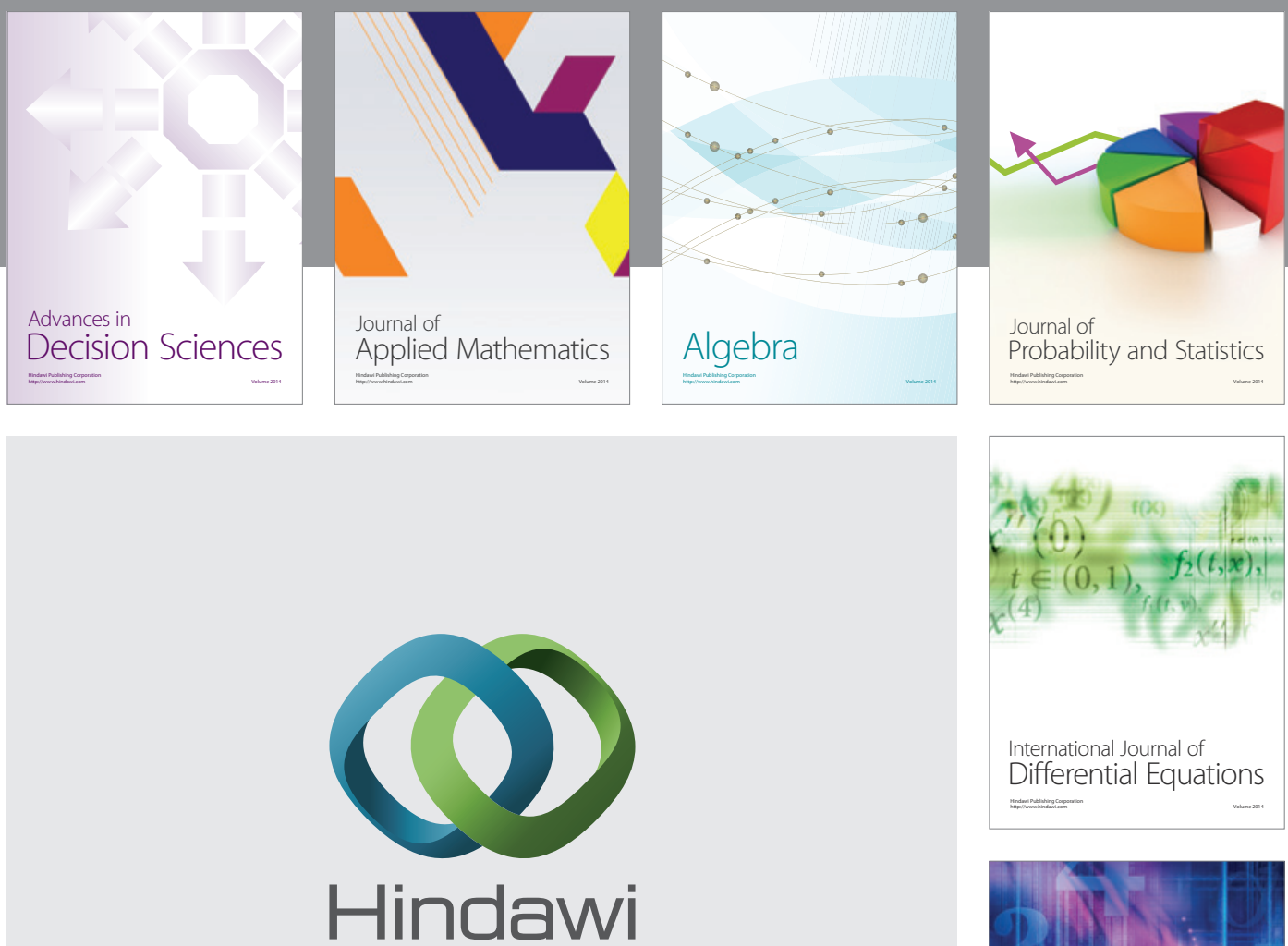

Submit your manuscripts at http://www.hindawi.com
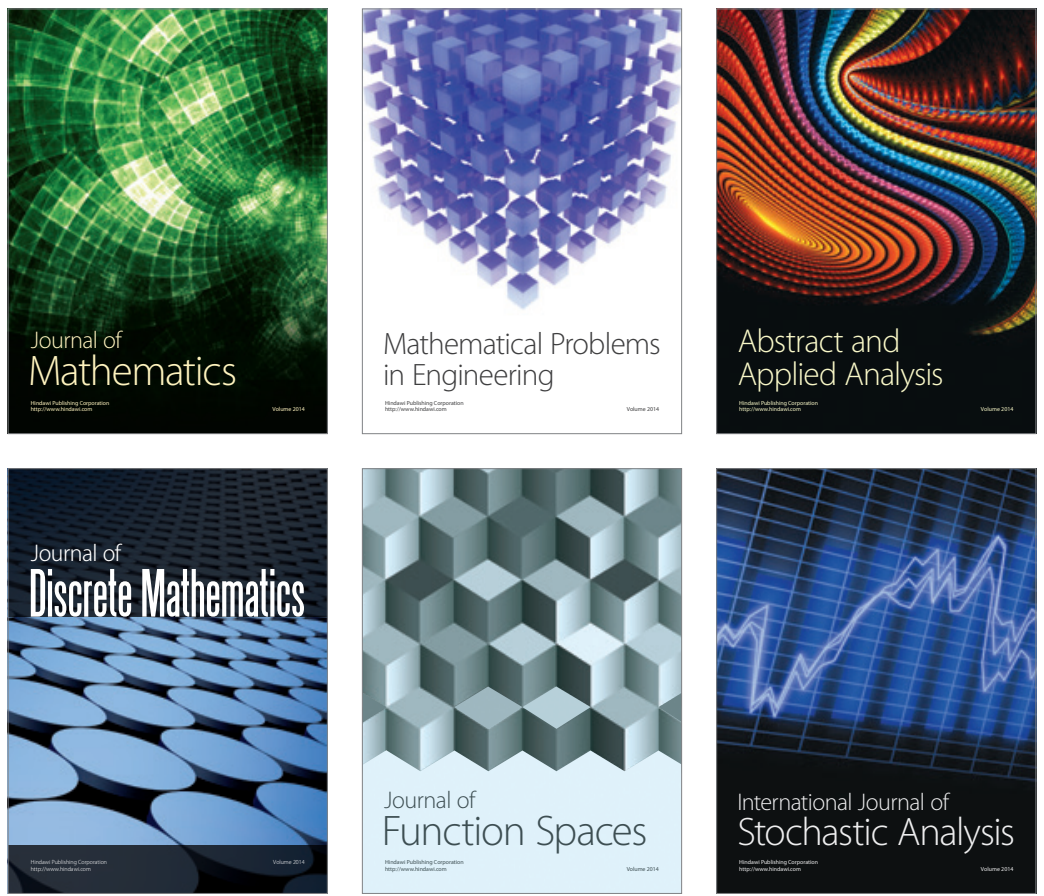

Journal of

Function Spaces

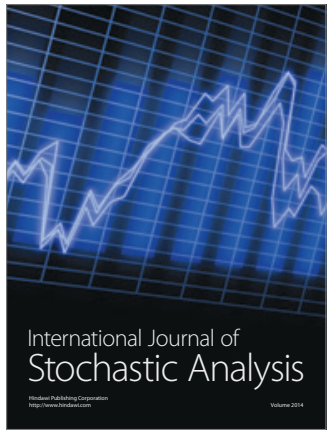

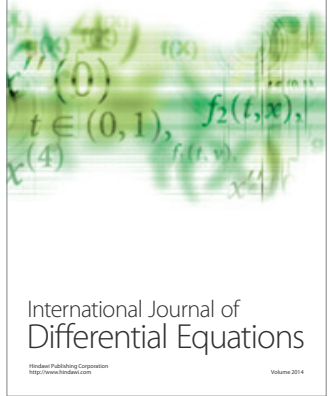
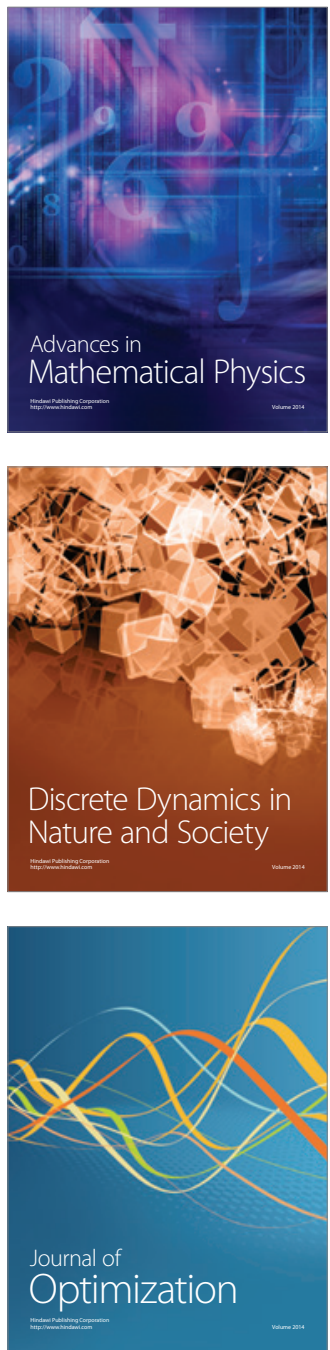